\title{
Degree powers in graphs with a forbidden even cycle
}

\author{
Vladimir Nikiforov* \\ Department of Mathematical Sciences, University of Memphis, \\ Memphis, TN 38152, USA, \\ vnikifrv@memphis.edu
}

Submitted: Dec 27, 2008; Accepted: Aug 11, 2009; Published: Aug 21, 2009

Mathematics Subject Classifications: 05C35, 05C38

\begin{abstract}
Let $C_{l}$ denote the cycle of length $l$. For $p \geqslant 2$ and integer $k \geqslant 1$, we prove that the function

$$
\phi(k, p, n)=\max \left\{\sum_{u \in V(G)} d^{p}(u): G \text { is a graph of order } n \text { containing no } C_{2 k+2}\right\}
$$

satisfies $\phi(k, p, n)=k n^{p}(1+o(1))$. This settles a conjecture of Caro and Yuster. Our proof is based on a new sufficient condition for long paths.
\end{abstract}

\section{Introduction}

Our notation and terminology follow [1]; in particular, $C_{l}$ denotes the cycle of length $l$.

For $p \geqslant 2$ and integer $k \geqslant 1$, Caro and Yuster [3], among other things, studied the function

$$
\phi(k, p, n)=\max \left\{\sum_{u \in V(G)} d_{G}^{p}(u): G \text { is a graph of order } n \text { without a } C_{2 k+2}\right\}
$$

and conjectured that

$$
\phi(k, p, n)=k n^{p}(1+o(1)) .
$$

The graph $K_{k}+\bar{K}_{n-k}$, i.e., the join of $K_{k}$ and $\bar{K}_{n-k}$, gives $\phi(k, p, n)>k(n-1)^{p}$, so to prove (1), a matching upper bound is necessary. We give such a bound in Corollary 3 below. Our main tool, stated in Lemma 1, is a new sufficient condition for long paths. This result has other applications as well, for instance, the following spectral bound, proved in [5]:

\footnotetext{
*This research has been supported by NSF Grant \# DMS-0906634.
} 
Let $G$ be a graph of order $n$ and $\mu$ be the largest eigenvalue of its adjacency matrix. If $G$ does not contain $C_{2 k+2}$, then

$$
\mu^{2}-k \mu \leqslant k(n-1)
$$

\section{Main results}

We write $|X|$ for the cardinality of a finite set $X$. Let $G$ be a graph, and $X$ and $Y$ be disjoint sets of vertices of $G$. We write:

- $V(G)$ for the vertex set of $G$ and $|G|$ for $|V(G)|$;

- $e_{G}(X)$ for the number of edges induced by $X$;

- $e_{G}(X, Y)$ for the number of edges joining vertices in $X$ to vertices in $Y$;

- $G-u$ for the graph obtained by removing the vertex $u \in V(G)$;

- $\Gamma_{G}(u)$ for the set of neighbors of the vertex $u$ and $d_{G}(u)$ for $\left|\Gamma_{G}(u)\right|$.

The main result of this note is the following lemma.

Lemma 1 Suppose that $k \geqslant 1$ and let the vertices of a graph $G$ be partitioned into two sets $A$ and $B$.

(1) If

$$
2 e_{G}(A)+e_{G}(A, B)>(2 k-2)|A|+k|B|,
$$

then there exists a path of order $2 k$ or $2 k+1$ with both ends in $A$.

(2) If

$$
2 e_{G}(A)+e_{G}(A, B)>(2 k-1)|A|+k|B|,
$$

then there exists a path of order $2 k+1$ with both ends in $A$.

Note that if we choose the set $B$ to be empty, Lemma 1 amounts to a classical result of Erdős and Gallai:

If a graph of order $n$ has more than $k n / 2$ edges, then it contains a path of order $k+2$.

We postpone the proof of Lemma 1 to Section 3 and turn to two consequences.

Theorem 2 Let $G$ be a graph with $n$ vertices and $m$ edges. If $G$ does not contain a $C_{2 k+2}$, then

$$
\sum_{u \in V(G)} d_{G}^{2}(u) \leqslant 2 k m+k(n-1) n .
$$

Proof Let $u$ be any vertex of $G$. Partition the vertices of the graph $G-u$ into the sets $A=\Gamma_{G}(u)$ and $B=V(G) \backslash\left(\Gamma_{G}(u) \cup\{u\}\right)$. Since $G$ contains no $C_{2 k+2}$, the graph $G-u$ does not contain a path of order $2 k+1$ with both ends in $A$. Applying Lemma 1, part (2), we see that

$$
2 e_{G-u}(A)+e_{G-u}(A, B) \leqslant(2 k-1)|A|+k|B|,
$$


and therefore,

$$
\begin{aligned}
\sum_{v \in \Gamma_{G}(u)}\left(d_{G}(v)-1\right) & =\sum_{v \in \Gamma_{G}(u)} d_{G-u}(v)=2 e_{G-u}(A)+e_{G-u}(A, B) \\
& \leqslant(2 k-1)|A|+k|B| \\
& =(2 k-1) d_{G}(u)+k\left(n-d_{G}(u)-1\right) .
\end{aligned}
$$

Rearranging both sides, we obtain

$$
\sum_{v \in \Gamma_{G}(u)} d_{G}(v) \leqslant k d_{G}(u)+k(n-1)
$$

Adding these inequalities for all vertices $u \in V(G)$, we find out that

$$
\sum_{u \in V(G)} \sum_{v \in \Gamma_{G}(u)} d_{G}(v) \leqslant k \sum_{u \in V(G)} d_{G}(u)+k(n-1) n=2 k m+k(n-1) n .
$$

To complete the proof of the theorem note that the term $d_{G}(v)$ appears in the left-hand sum exactly $d_{G}(v)$ times, and so

$$
\sum_{u \in V(G)} \sum_{v \in \Gamma_{G}(u)} d_{G}(v)=\sum_{v \in V(G)} d_{G}^{2}(v) .
$$

Here is a corollary of Theorem 2 that gives the upper bound for the proof of (1).

Corollary 3 Let $G$ be a graph with $n$ vertices. If $G$ does not contain a $C_{2 k+2}$, then for every $p \geqslant 2$,

$$
\sum_{u \in V(G)} d_{G}^{p}(u) \leqslant k n^{p}+O\left(n^{p-1 / 2}\right) .
$$

Proof Letting $m$ be the number of edges of $G$, we first deduce an upper bound on $m$. Theorem 2 and the AM-QM inequality imply that

$$
\frac{4 m^{2}}{n} \leqslant \sum_{u \in V(G)} d_{G}^{2}(u) \leqslant 2 k m+k(n-1) n,
$$

and so,

$$
m \leqslant-k n+n \sqrt{k(n-1)+k^{2}}<n \sqrt{k n} .
$$

Note that much stronger upper bounds on $m$ are known (e.g., see [2] and [6]), but this one is simple and unconditional.

Now Theorem 2 and inequality (4) imply that

$$
\begin{aligned}
\sum_{u \in V(G)} d_{G}^{p}(u) & <\sum_{u \in V(G)} n^{p-2} d_{G}^{2}(u)<k n^{p}+2 k m n^{p-2}<k n^{p}+2(k n)^{3 / 2} n^{p-2} \\
& =k n^{p}+O\left(n^{p-1 / 2}\right)
\end{aligned}
$$

completing the proof.

Note that we need only part (2) of Lemma 1 to prove Theorem 2 and Corollary 3. However, part (1) of Lemma 1 may have also applications, as shown in [5]. 


\section{Proof of Lemma 1}

To simplify the proof of Lemma 1 we state two routine lemmas whose proofs are omitted.

Lemma 4 Let $P=\left(v_{1}, \ldots, v_{p}\right)$ be a path of maximum order in a connected non-Hamiltonian graph $G$. Then $p \geqslant d_{G}\left(v_{1}\right)+d_{G}\left(v_{p}\right)+1$.

Lemma 5 Let $P=\left(v_{1}, \ldots, v_{p}\right)$ be a path of maximum order in a graph $G$. Then either $v_{1}$ is joined to two consecutive vertices of $P$ or $G$ contains a cycle of order at least $2 d_{G}\left(v_{1}\right)$.

Proof of Lemma 1 For convenience we shall assume that the set $B$ is independent. Also, we shall call a path with both ends in $A$ an $A$-path.

Claim 6 If $G$ contains an A-path of order $p>2$, then $G$ contains an A-path of order $p-2$.

Indeed, let $\left(v_{1}, \ldots, v_{p}\right)$ be an $A$-path. If $v_{2} \in B$, then $v_{3} \in A$, and so $\left(v_{3}, \ldots, v_{p}\right)$ is an $A$-path of order $p-2$. If $v_{p-1} \in B$, then $v_{p-2} \in A$, and so $\left(v_{1}, \ldots, v_{p-2}\right)$ is an $A$-path of order $p-2$. Finally, if both $v_{2} \in A$ and $v_{p-1} \in A$, then $\left(v_{2}, \ldots, v_{p-1}\right)$ is an $A$-path of order $p-2$.

The proofs of the two parts of Lemma 1 are very similar, but since they differ in the details, we shall present them separately.

\section{Proof of part (1)}

From Claim 6 we easily obtain the following consequence:

Claim 7 If $G$ contains an A-path of order $p \geqslant 2 k$, then $G$ contains an A-path of order $2 k$ or $2 k+1$.

This in turn implies

Claim 8 If $G$ contains a cycle $C_{p}$ for some $p \geqslant 2 k+1$, then $G$ contains an A-path of order $2 k$ or $2 k+1$.

Indeed, let $C=\left(v_{1}, \ldots, v_{p}, v_{1}\right)$ be a cycle of order $p \geqslant 2 k+1$. The assertion is obvious if $C$ is entirely in $A$, so let assume that $C$ contains a vertex of $B$, say $v_{1} \in B$. Then $v_{2} \in A$ and $v_{p} \in A$; hence, $\left(v_{2}, \ldots, v_{p}\right)$ is an $A$-path of order at least $2 k$. In view of Claim 7 , this completes the proof of Claim 8.

To complete the proof of part (1) we shall use induction on the order of $G$. First we show that condition (2) implies that $|G| \geqslant 2 k$. Indeed, assume that $|G| \leqslant 2 k-1$. We have

$$
|A|^{2}-|A|+|A||B| \geqslant 2 e_{G}(A)+e_{G}(A, B)>(2 k-2)|A|+k|B|
$$

and so,

$$
|G|(|A|-k)=(|A|+|B|)(|A|-k)>(k-1)|A| .
$$


Hence, we find that

$$
(2 k-1)(|A|-k)>(k-1)|A|
$$

and so, $|A|>2 k-1$, a contradiction with $|A| \leqslant|G|$.

The conclusion of Lemma 1 , part (1) follows when $|G| \leqslant 2 k-1$ since then the hypothesis is false. Assume now that $|G| \geqslant 2 k$ and that the Lemma holds for graphs with fewer vertices than $G$. It is easy to see that this assumption implies the assertion if $G$ is disconnected. Indeed, let $G_{1}, \ldots, G_{s}$ be the components of $G$. Assuming that $G$ has no $A$-path of order $2 k+1$, the inductive assumption implies that each component $G_{i}$ satisfies

$$
2 e_{G_{i}}\left(A_{i}\right)+e_{G_{i}}\left(A_{i}, B_{i}\right) \leqslant(2 k-2)\left|A_{i}\right|+k\left|B_{i}\right|,
$$

where

$$
A_{i}=A \cap V\left(G_{i}\right) \quad \text { and } \quad B_{i}=B \cap V\left(G_{i}\right) .
$$

Summing (5) for $i=1, \ldots, s$, we obtain a contradiction to (2).

Thus, to the end of the proof we shall assume that $G$ is connected. Also, we can assume that $G$ is non-Hamiltonian. Indeed, in view of Claim 8, this is obvious when $|G|>2 k$. If $|G|=2 k$ and $G$ is Hamiltonian, then no two consecutive vertices along the Hamiltonian cycle belong to $A$, and since $B$ is independent, we have $|B|=|A|=k$. Then

$$
k(2 k-1) \geqslant 2 e_{G}(A)+e_{G}(A, B)>(2 k-2)|A|+k|B|=k(2 k-1),
$$

contradicting (2). Thus, we shall assume that $G$ is non-Hamiltonian.

The induction step is completed if there is a vertex $u \in B$ such that $d_{G}(u) \leqslant k$. Indeed the sets $A$ and $B^{\prime}=B \backslash\{u\}$ partition the vertices of $G-u$ and also

$$
\begin{aligned}
2 e_{G-u}(A)+e_{G-u}(A, B) & =2 e_{G}(A)+e_{G}(A, B)-d_{G}(u)>(2 k-2)|A|+k|B|-k \\
& =(2 k-2)|A|+k\left|B^{\prime}\right|
\end{aligned}
$$

hence $G-u$ contains an $A$-path of order at least $2 k$, completing the proof. Thus, to the end of the proof we shall assume that

(a) $d_{G}(u) \geqslant k+1$ for every vertex $u \in B$.

For every vertex $u \in A$, write $d_{G}^{\prime}(u)$ for its neighbors in $A$ and $d_{G}^{\prime \prime}(u)$ for its neighbors in $B$. The induction step can be completed if there is a vertex $u \in A$ such that $2 d_{G}^{\prime}(u)+$ $d_{G}^{\prime \prime}(u) \leqslant 2 k-2$. Indeed, if $u$ is such a vertex, note that the sets $A^{\prime}=A \backslash\{u\}$ and $B$ partition the vertices of $G-u$ and also

$$
\begin{aligned}
2 e_{G-u}(A)+e_{G-u}(A, B) & =2 e_{G}(A)+e_{G}(A, B)-2 d_{G}^{\prime}(u)-d_{G}^{\prime \prime}(u) \\
& >(2 k-2)|A|+k|B|-2 k+2 \\
& =(2 k-2)\left|A^{\prime}\right|+k|B| ;
\end{aligned}
$$

hence $G-u$ contains an $A$-path of order at least $2 k$, completing the proof. Hence we have $2 d_{G}^{\prime}(u)+d_{G}^{\prime \prime}(u) \geqslant 2 k-1$, and so $d_{G}(u) \geqslant k$. Thus, to the end of the proof, we shall assume that: 
(b) $d_{G}(u) \geqslant k$ for every vertex $u \in A$.

Select now a path $P=\left(v_{1}, \ldots, v_{p}\right)$ of maximum length in $G$. To complete the induction step we shall consider three cases: (i) $v_{1} \in B, v_{p} \in B$; (ii) $v_{1} \in B, v_{p} \in A$, and (iii) $v_{1} \in A, v_{p} \in A$.

Case (i): $v_{1} \in B, v_{p} \in B$

In view of assumption $(a)$ we have $d_{G}\left(v_{1}\right)+d_{G}\left(v_{p}\right) \geqslant 2 k+2$, and Lemma 4 implies that $p \geqslant 2 k+3$. We see that $\left(v_{2}, \ldots, v_{p-1}\right)$ is an $A$-path of order at least $2 k+1$, completing the proof by Claim 7 .

Case (ii): $v_{1} \in B, v_{p} \in A$

In view of assumptions $(a)$ and $(b)$ we have $d_{G}\left(v_{1}\right)+d_{G}\left(v_{p}\right) \geqslant 2 k+1$, and Lemma 4 implies that $p \geqslant 2 k+2$, and so, $\left(v_{2}, \ldots, v_{p}\right)$ is an $A$-path of order at least $2 k+1$. This completes the proof by Claim 7 .

Case (iii): $v_{1} \in A, v_{p} \in A$

In view of assumption (b) we have $d_{G}\left(v_{1}\right)+d_{G}\left(v_{p}\right) \geqslant 2 k$, and Lemma 4 implies that $p \geqslant 2 k+1$. Since $\left(v_{1}, \ldots, v_{p}\right)$ is an $A$-path of order at least $2 k+1$, by Claim 7 , the proof of part (A) of Lemma 1 is completed.

\section{Proof of part (2)}

From Claim 6 we easily obtain the following consequence:

Claim 9 If $G$ contains an A-path of odd order $p \geqslant 2 k+1$, then $G$ contains an A-path of order exactly $2 k+1$.

From Claim 9 we deduce another consequence:

Claim 10 If $G$ contains a cycle $C_{p}$ for some $p \geqslant 2 k+1$, then $G$ contains an A-path of order exactly $2 k+1$.

Indeed, let $C=\left(v_{1}, \ldots, v_{p}, v_{1}\right)$ be a cycle of order $p \geqslant 2 k+1$. If $p$ is odd, then some two consecutive vertices of $C$ belong to $A$, say the vertices $v_{1}$ and $v_{2}$. Then $\left(v_{2}, \ldots, v_{p}, v_{1}\right)$ is an $A$-path of odd order $p \geqslant 2 k+1$, and by Claim 9 the assertion follows. If $p$ is even, then $p \geqslant 2 k+2$. The assertion is obvious if $C$ is entirely in $A$, so let assume that $C$ contains a vertex of $B$, say $v_{1} \in B$. Then $v_{2} \in A$ and $v_{p} \in A$; hence $\left(v_{2}, \ldots, v_{p}\right)$ is an $A$-path of odd order at least $2 k+1$, completing the proof of Claim 10 .

To complete the proof of Lemma 1 we shall use induction on the order of $G$. First we show that condition (3) implies that $|G| \geqslant 2 k+1$. Indeed, assume that $|G| \leqslant 2 k$. We have

$$
|A|^{2}-|A|+|A||B| \geqslant 2 e_{G}(A)+e_{G}(A, B)>(2 k-1)|A|+k|B|
$$

and so,

$$
|G|(|A|-k)=(|A|+|B|)(|A|-k)>k|A| .
$$


Hence, we find that $2 k(|A|-k)>k|A|$, and $|A|>2 k$, contradicting that $|A| \leqslant|G|$.

The conclusion of Lemma 1, part (2) follows when $|G| \leqslant 2 k$ since then the hypothesis is false. Assume now that $|G| \geqslant 2 k+1$ and that the assertion holds for graphs with fewer vertices than $G$. As in part (1), it is easy to see that this assumption implies the assertion if $G$ is disconnected, so to the end of the proof we shall assume that $G$ is connected. Also, in view of Claim 10 and $|G| \geqslant 2 k+1$, we shall assume that $G$ is non-Hamiltonian.

The induction step is completed if there is a vertex $u \in B$ such that $d_{G}(u) \leqslant k$. Indeed the sets $A$ and $B^{\prime}=B \backslash\{u\}$ partition the vertices of $G-u$ and also

$$
\begin{aligned}
2 e_{G-u}(A)+e_{G-u}(A, B) & =2 e_{G}(A)+e_{G}(A, B)-d_{G}(u) \\
& >(2 k-1)|A|+k|B|-k \\
& =(2 k-1)|A|+k\left|B^{\prime}\right|
\end{aligned}
$$

hence $G-u$ contains an $A$-path of order $2 k+1$, completing the proof. Thus, to the end of the proof we shall assume that:

(a) $d_{G}(u) \geqslant k+1$ for every vertex $u \in B$.

For every vertex $u \in A$, write $d_{G}^{\prime}(u)$ for its neighbors in $A$ and $d_{G}^{\prime \prime}(u)$ for its neighbors in $B$. The induction step can be completed if there is a vertex $u \in A$ such that $2 d_{G}^{\prime}(u)+$ $d_{G}^{\prime \prime}(u) \leqslant 2 k-1$. Indeed, if $u$ is such a vertex, note that the sets $A^{\prime}=A \backslash\{u\}$ and $B$ partition the vertices of $G-u$ and also

$$
\begin{aligned}
2 e_{G-u}(A)+e_{G-u}(A, B) & =2 e_{G}(A)+e_{G}(A, B)-2 d_{G}^{\prime}(u)-d_{G}^{\prime \prime}(u) \\
& >(2 k-1)|A|+k|B|-2 k+1 \\
& =(2 k-1)\left|A^{\prime}\right|+k|B|
\end{aligned}
$$

hence $G-u$ contains an $A$-path of order $2 k+1$, completing the proof. Thus, to the end of the proof, we shall assume that:

(b) $d_{G}(u) \geqslant k$ for every vertex $u \in A$ and if $u$ has neighbors in $B$, then $d_{G}(u) \geqslant k+1$.

Select now a path $P=\left(v_{1}, \ldots, v_{p}\right)$ of maximum length in $G$. To complete the induction step we shall consider three cases: (i) $v_{1} \in B, v_{p} \in B$; (ii) $v_{1} \in B, v_{p} \in A$, and (iii) $v_{1} \in A, v_{p} \in A$.

Case (i): $v_{1} \in B, v_{p} \in B$

In view of assumption (b) we have $d_{G}\left(v_{1}\right)+d_{G}\left(v_{p}\right) \geqslant 2 k+2$, and Lemma 4 implies that $p \geqslant 2 k+3$. If $p$ is odd, we see that $\left(v_{2}, \ldots, v_{p-1}\right)$ is an $A$-path of order at least $2 k+1$, and by Claim 9, the proof is completed.

Suppose now that $p$ is even. Applying Lemma 5, we see that either $G$ has a cycle of order at least $2 d_{G}\left(v_{1}\right) \geqslant 2 k+2$, or $v_{1}$ is joined to $v_{i}$ and $v_{i+1}$ for some $i \in\{2, \ldots, p-2\}$. In the first case we complete the proof by Claim 10; in the second case we see that the sequence

$$
\left(v_{2}, v_{3}, \ldots, v_{i}, v_{1}, v_{i+1}, v_{i+2}, \ldots, v_{p-1}\right)
$$


is an $A$-path of order $p-1$. Since $p-1$ is odd and $p-1 \geqslant 2 k+3$, the proof is completed by Claim 9 .

Case (ii): $v_{1} \in B, v_{p} \in A$

In view of assumptions $(a)$ and $(b)$ we have $d_{G}\left(v_{1}\right)+d_{G}\left(v_{p}\right) \geqslant 2 k+1$, and Lemma 4 implies that $p \geqslant 2 k+2$. If $p$ is even, we see that $\left(v_{2}, \ldots, v_{p-1}\right)$ is an $A$-path of order at least $2 k+1$, and by Claim 9, the proof is completed.

Suppose now that $p$ is odd. Applying Lemma 5, we see that either $G$ has a cycle of order at least $2 d_{G}\left(v_{1}\right) \geqslant 2 k+2$, or $v_{1}$ is joined to $v_{i}$ and $v_{i+1}$ for some $i \in\{2, \ldots, p-1\}$. In the first case we complete the proof by Claim 10; in the second case we see that the sequence

$$
\left(v_{2}, v_{3}, \ldots, v_{i}, v_{1}, v_{i+1}, v_{i+2}, \ldots, v_{p}\right)
$$

is an $A$-path of order $p$. Since $p$ is odd and $p \geqslant 2 k+2$, the proof is completed by Claim 9 .

Case (iii): $v_{1} \in A, v_{p} \in A$

In view of assumption (b) we have $d_{G}\left(v_{1}\right)+d_{G}\left(v_{p}\right) \geqslant 2 k$, and Lemma 4 implies that $p \geqslant 2 k+1$. If $p$ is odd, the proof is completed by Claim 9 .

Suppose now that $p$ is even, and therefore, $p \geqslant 2 k+2$. If $v_{2} \in A$, then the sequence $\left(v_{2}, \ldots, v_{p}\right)$ is an $A$-path of odd order $p-1 \geqslant 2 k+1$, completing the proof by Claim 9 . If $v_{2} \in B$, we see that $v_{1}$ has a neighbor in $B$, and so, $d_{G}\left(v_{1}\right) \geqslant k+1$.

Applying Lemma 5, we see that either $G$ has a cycle of order at least $2 d_{G}\left(v_{1}\right) \geqslant 2 k+2$, or $v_{1}$ is joined to $v_{i}$ and $v_{i+1}$ for some $i \in\{2, \ldots, p-2\}$. In the first case we complete the proof by Claim 10. In the second case we shall exhibit an $A$-path of order $p-1$. Indeed, if $i=2$, let

$$
Q=\left(v_{1}, v_{3}, v_{4}, \ldots, v_{p}\right)
$$

and if $i \geqslant 3$, let

$$
Q=\left(v_{3}, \ldots, v_{i}, v_{1}, v_{i+1}, v_{i+2}, \ldots, v_{p}\right) .
$$

In either case $Q$ is an $A$-path of order $p-1$. Since $p-1$ is odd and $p-1 \geqslant 2 k+1$, the proof is completed by Claim 9 .

This completes the proof of Lemma 1.

Acknowledgment Thanks are due to Dick Schelp and Ago Riet for useful discussions on Lemma 1.

\section{References}

[1] B. Bollobás, Modern Graph Theory, Graduate Texts in Mathematics, 184, SpringerVerlag, New York (1998), xiv+394 pp.

[2] J. A. Bondy and M. Simonovits, Cycles of even length in graphs, J. Comb. Theory Ser. B 16 (1974), 97-105. 
[3] Y. Caro, R. Yuster, A Turán type problem concerning the powers of the degrees of a graph, Electron. J. Comb. 7 (2000), RP 47.

[4] P. Erdős, T. Gallai, On maximal paths and circuits of graphs, Acta Math. Acad. Sci. Hungar. 10 (1959), 337-356.

[5] V. Nikiforov, The spectral radius of graphs without paths and cycles of specified length, to appear in Linear Algebra Appl. Preprint available at arXiv:0903.535

[6] J. Verstraëte, On arithmetic progressions of cycle lengths in graphs, Combin. Probab. Comput. 9 (2000), 369-373. 